\title{
Could Estrogen Protect Women From COVID-19?
}

\author{
Roxanna Pirhadia , Vikram Sinai Talaulikar ${ }^{\mathrm{b},}$ e, Joseph Onwude ${ }^{\mathrm{c}}$, Isaac Manyonda ${ }^{\mathrm{d}}$
}

\begin{abstract}
The apparent gender differences in favor of women in the risk of contracting and dying from coronavirus disease 2019 (COVID-19), and the fact that such trends have also been observed in recent epidemics including severe acute respiratory syndrome (SARS) and Middle East respiratory syndrome (MERS), have prompted the obvious question: Are the reasons life-style or biological? True, women generally make healthier lifestyle choices as compared to men. Women do not smoke or drink as much as men, and they have a lower burden of those diseases (heart disease, diabetes or chronic lung conditions) that are known to be significant factors in the higher death rates among men with COVID-19. But there is compelling evidence for a role for biological factors. Genes are likely to play an important role. The X chromosome, of which women possess two, contains the largest number of immunerelated genes of the whole human genome, theoretically giving women double the advantage over men in mounting an efficient and rapid immune response. A fundamental difference between women and men is their hormonal milieu, and it is not unreasonable to suppose that the dominant female hormone estrogen could influence the response to infection. In this paper we evaluate the evidence and mechanisms by which estrogen could provide protection to women from a variety of viruses, perhaps including the coronavirus that causes COVID-19.
\end{abstract}

Keywords: COVID-19; Coronavirus; Infection; Estrogen; Protection

\section{Introduction}

It is generally accepted that the coronavirus that causes the

Manuscript submitted July 26, 2020, accepted September 1, 2020

Published online September 21, 2020

aFaculty of Health, Education, Medicine and Social Care, Anglia Ruskin University, Second Floor William Harvey Building, Bishop Hall Lane, Chelmsford CM1 1SQ, UK

bReproductive Medicine Unit, EGA Wing, University College London Hospital, 235 Euston Road, London NW1 2BU, UK

${ }^{c}$ The Chelmsford Private Day Surgery Hospital, Fenton House, 85-89 New London Road, Chelmsford CM2 OPP, UK

dDepartment of Obstetrics and Gynaecology, St George's University Hospitals NHS Foundation Trust/St George's, University of London, London, UK ${ }^{\mathrm{e}}$ Corresponding Author: Vikram Sinai Talaulikar, Reproductive Medicine Unit, EGA Wing, University College London Hospital, 235 Euston Road, London NW1 2BU, UK. Email: vikram.talaulikar@nhs.net

doi: https://doi.org/10.14740/jocmr4303 new coronavirus disease 2019 (COVID-19) pandemic infects and kills more men than women [1]. A sex and gender dichotomy on the impact of epidemic-prone infections has long been recognized by the World Health Organization (WHO) [2]. Studies investigating the role of gender in morbidity and mortality in patients with COVID-19 have shown that men with COVID-19 are more at risk for worse outcomes and death, independent of age $[1,3]$. Men tend to develop more serious infections than women and the number of men is about 2.4 times that of women in the deceased patients [3]. Sex-disaggregated data from 26 countries indicate that the overall case fatality ratio in men is higher than that in women [4].

Virus infections in particular appear to have a stronger predilection for men, and this was observed in recent epidemics including the severe acute respiratory syndrome (SARS) [5] and Middle East respiratory syndrome (MERS) [6]. The reasons for these female-male differences are not fully understood, but they are likely to involve interplay between lifestyle factors and biological influences. From a life-style perspective, women tend to drink and smoke less than men, and consequently often have a lower burden of those diseases, such as cardiovascular disease, diabetes and chronic lung disease, which appear to increase mortality risk in COVID-19 patients [7]. However, biological influences could be even more important. The immune response is likely to play a critical role, and it is generally known that there are gender differences in immune responsiveness [8]. Within the whole human genome, the $\mathrm{X}$ chromosome contains the largest number of immune-related genes [9], and women possess two of these chromosomes versus one in men. In 2008, a list of 79 X-linked genes with a possible role in sex-based differences in immune responses was presented [10]. Some of these include ELK1, EPAG, GTD, $N E M O$ and $C Y B B$ in addition to immune response related proteins, receptors and transcriptional and translational control receptors.

It is teleologically sound to suppose that any difference in immune responsiveness between men and women is likely to be mediated through hormonal mechanisms, with the dominant female hormone estrogen playing a central role. If estrogen modulates the immune system, an obvious human model is a comparison of such responses between men and premenopausal women (estrogen replete) and postmenopausal women (naturally estrogen deficient), in particular studying the impact of estrogen supplementation in the latter. A wealth of data from such research is now available.

While estrogen is often thought of in terms of its key role in normal sexual development and reproduction, and while there is evidence of its immunomodulatory activities de- 


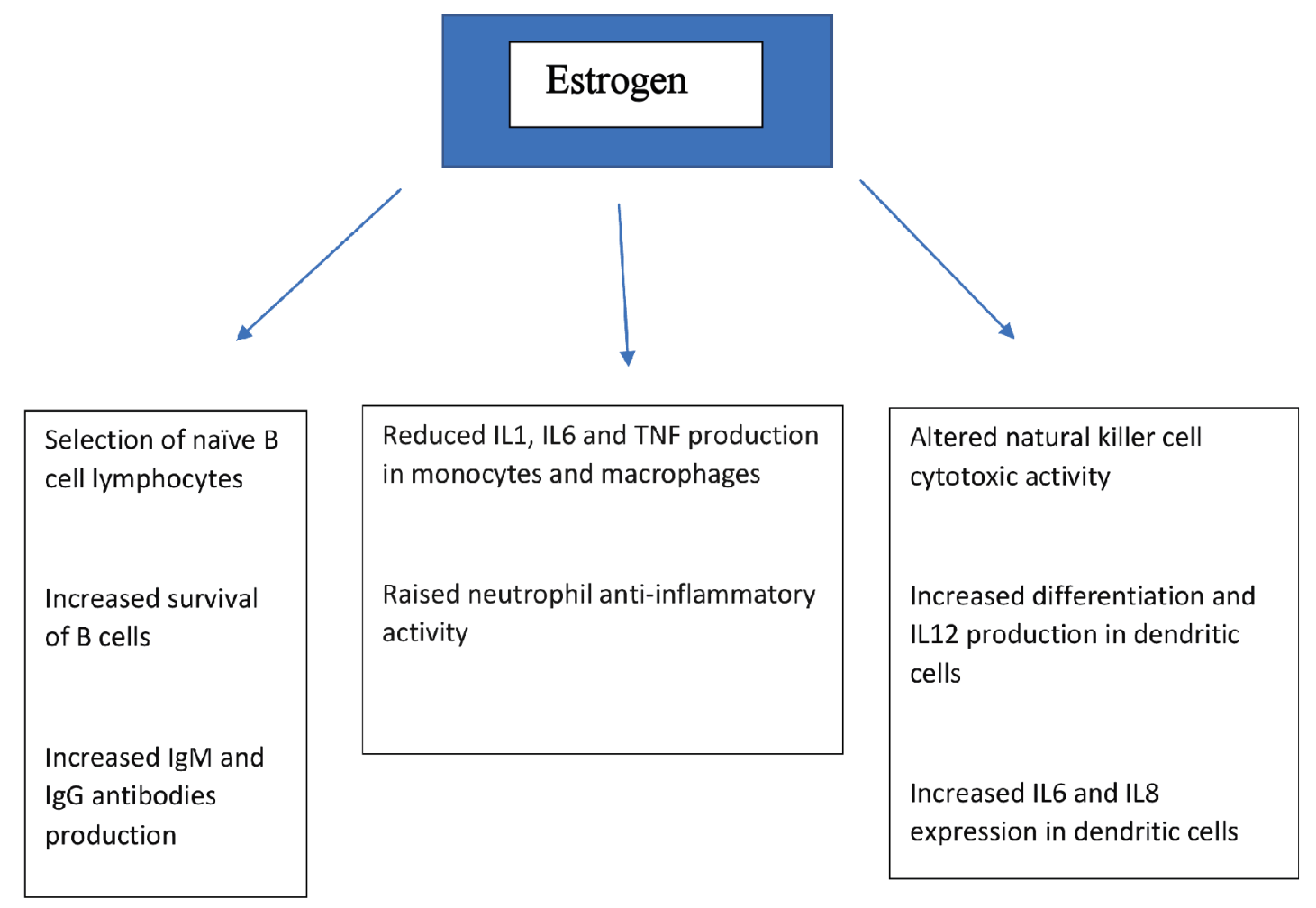

Figure 1. Effects of estrogen on various components of immune system. IL: interleukin; TNF: tumor necrosis factor; Ig: immunoglobulin.

scribed above, the presence of estrogen receptors in a variety of other body tissues and organ systems (including endometrium, breast, ovarian stromal and granulosa cells, hypothalamus, hippocampus, kidney, bone, heart, blood vessels, lungs, intestinal mucosa, prostate and endothelial cells) suggests that estrogen plays an important role in a wide range of physiological processes including immune response [11], by a variety of mechanisms that include direct action on relevant cell types/ tissue. Figure 1 shows the effect of estrogen on various components of the immune system. There are data from in vitro studies, animal experimentation and human research that support this contention. We searched the Medline/PubMed medical literature database using terms coronavirus, COVID-19, estrogen, estrogen, viral, infections and immunity to search for scientific manuscripts related to our topic, and here we explore some of the evidence and discuss the underlying mechanisms as they might relate to COVID-19 infection.

\section{Antiviral Actions of Estrogen: Effect on Innate Immunity and Non-Immune Systems}

Several lines of research have shown that estrogen exhibits antiviral properties. Smith et al [12] (2000) compared the influence of estrogen versus progesterone on the vaginal transmission of simian immunodeficiency virus. Six ovariectomized macaques were treated with estrogen, another six with progesterone, and a further six were untreated, followed by intra- vaginal inoculation with the virus. All six untreated (control) macaques and five $(83 \%)$ progesterone-treated animals became infected following intravaginal inoculation, while none of the six estrogen-treated macaques was infected. The block to infection occurred at the vaginal epithelium and/or lumen, since estrogen-treated macaques became infected following subepithelial inoculation of virus. Johansen et al [13] (2013) used molecular probes to identify drugs with antiviral activity against the Zaire Ebola virus. They identified a set of selective estrogen receptor modulators (SERMs), including clomiphene and toremifene, which acted as potent inhibitors of viral infection. Antiviral activity was confirmed for both of these SERMs in an in vivo mouse infection model. This antiviral activity occurred even in the absence of detectable estrogen receptor expression, and both SERMs inhibited virus entry after internalisation, suggesting that clomiphene and toremifene are not working through classical pathways associated with the estrogen receptor. Instead, the response appeared to be an offtarget effect where the compounds interfered with a step late in viral entry and triggering of fusion. Yet another study in 2017 analyzed the structures of SERMs and their incidental biological activity (cholesterol accumulation) as a plausible mechanism of their inhibitory effects on Ebola virus infection [14]. They demonstrated that the same dosages of SERMs which induced cholesterol accumulation also inhibited Ebola infection. SERMs reduced the cellular sphingosine and subsequently caused endolysosomal calcium accumulation, which in turn led to blocking the Ebola entry. 
Robinson et al [15] tested the hypothesis that estrogen protects against influenza A virus infection by altering the recruitment and activity of innate immune cells and $\mathrm{T}$ cells. Chemokine concentrations were measured, and innate and adaptive immune cells were enumerated from the lungs of estrogen and placebo-treated ovariectomized female C57BL/6 mice following infection. Females treated with estrogen experienced less morbidity but had similar lung virus titres to placebo-treated females. Females treated with estrogen had lower induction of CCL2 but higher CCL3 and CXCL1 responses in their lungs than placebo-treated females. Pulmonary recruitment of neutrophils, natural killer (NK) cells, macrophages, and dendritic cells was increased following infection, but only neutrophil numbers were greater in estrogentreated than placebo-treated females. Neutrophil depletion in estrogen-treated females increased morbidity, reduced pulmonary production of chemo-attractants for neutrophils, and reduced interferon production by virus-specific CD8 T cells. This study showed that neutrophils appear to mediate both inflammation and tissue repair during influenza virus infection and are regulated by estrogen to improve the outcome of infection in females [15].

In an in vitro study, Peretz et al [16] (2016) found that estrogenic compounds reduced influenza A virus replication in primary human nasal epithelial cells derived from females but not males [16]. These estrogenic compounds reduced peak viral titre, which did not involve changes in the secretion of chemokines or interferons, but rather was associated with reduced metabolic processes. The effects of estrogens were specific for cultures from female but not male donors. The antiviral effects were most likely mediated by signalling through estrogen receptors.

Antiviral effects of estrogens have also been reported previously against viruses such as human immunodeficiency virus (HIV), hepatitis C (HCV), Ebola and human cytomegalovirus (HCMV) [16]. Studies have shown that estradiol can prevent HIV transcription by blocking HIV promoter activity in peripheral blood mononuclear cells and preventing HIV entry in $\mathrm{CD}^{+} \mathrm{T}$ cell and macrophages $[17,18]$. Estradiol also prevents the production of infectious HCV particles [19]. SERMs, including clomiphene and raloxifene, inhibit HCV infection at multiple stages of the virus life cycle and act as adjuvants to improve antiviral HCV therapies in postmenopausal women $[16,20,21]$.

Tamoxifen (TAM) is an estrogen-receptor antagonist, and there was a study aimed to investigate the effect of TAM on infection with vesicular stomatitis virus (VSV) [22]. Vero cells (mice) were treated with different concentrations of TAM for $24 \mathrm{~h}$ and then infected with VSV. Additionally, mice were pretreated with $4 \mathrm{mg}$ TAM, 1 day and 3 days before infection with VSV. Treatment of Vero cells with TAM suppressed the viral replication of VSV in vitro and in vivo. The inhibitory effect of TAM on VSV replication correlated with an enhanced interferon response and stimulation of macrophages [22].

Estrogen treatment in ovariectomized animals reduces expression of vascular monocyte chemoattractant protein-1 (MCP-1) and leukocyte infiltration into injured tissues, such as arteries and lung [23, 24]. Estrogen is also able to modulate the activation of different macrophage immune phenotypes [25].

\section{Estrogen Modulates the Adaptive Immune Sys- tem}

The human immune system demonstrates significant sex differences. Women tend to have a more responsive immune system compared to men so that the outcome and survival rates from infections or sepsis are often better in females than in males [26].

We investigated the effect of therapeutic doses of $17 \beta$-estradiol on cellular immune responses in postmenopausal women [27]. Fifteen healthy women who had undergone a natural menopause were treated with estrogen in the form of $100 \mathrm{mg}$ patches applied twice weekly for 3 out of every 4 weeks over a 3-month period, followed by combined estrogen and progestogen formulations as long-term therapy.

Blood samples were taken on two occasions prior to treatment and at weeks 1, 3, 4, 7, 9, 12 and 24 after commencing therapy. Lymphocyte subsets (CD2, CD4, CD8, CD19, human leukocyte antigen-DR (HLA-DR) and NK) were studied in each blood sample using a monoclonal antibody kit and a twocolor fluorescence flow cytometer. One-way mixed lymphocyte reactions (MLRs) were performed using the same stimulator throughout. Delayed hypersensitivity skin tests (DHTs) were carried out twice before treatment and at weeks 3, 4, 12 and 24. Lymphocyte subsets did not change significantly with treatment, but both the MLRs and the DHTs were significantly depressed, maximally so by the third week of treatment showing that therapeutic doses of estrogen modulate certain immune responses. All these observations certainly confirm an interaction between the endocrine and immune systems. The hormonal milieu undoubtedly influences the immune system and sex steroids in particular appear to be potent modulators of the immune response [27].

In a 2016 study the effect of estrogen therapy on lymphocyte subset frequency and the immune response to influenza vaccine in three groups (younger women with regular menstrual cycles, not on hormonal contraception; postmenopausal women not on hormone replacement therapy; and postmenopausal hysterectomized women on estrogen-only replacement) were studied. The results suggested that humoral immunity is increased by estrogen therapy [28]. A summary of observational studies evaluating effects of estrogen on immune response is presented in Table 1 [12-17, 19-25, 27, 28].

Numerous additional studies, beyond the scope of this article, have confirmed male-female differences in the immune response, that generally the female immune response is superior, and estrogen driven, but also that this superior response could explain the higher prevalence of auto-immune disease in women when the immune system behaves in an aberrant fashion [29-33]. An age relationship between estrogen levels and monocyte/macrophage number and function has also been identified, with studies suggesting impaired macrophage function in postmenopausal women [34].

\section{Estrogen Protects Cardiovascular Health}

Cardiovascular complications are known to be a factor in a 
Table 1. Summary of Observational Studies Evaluating Effects of Estrogen on Immune Response

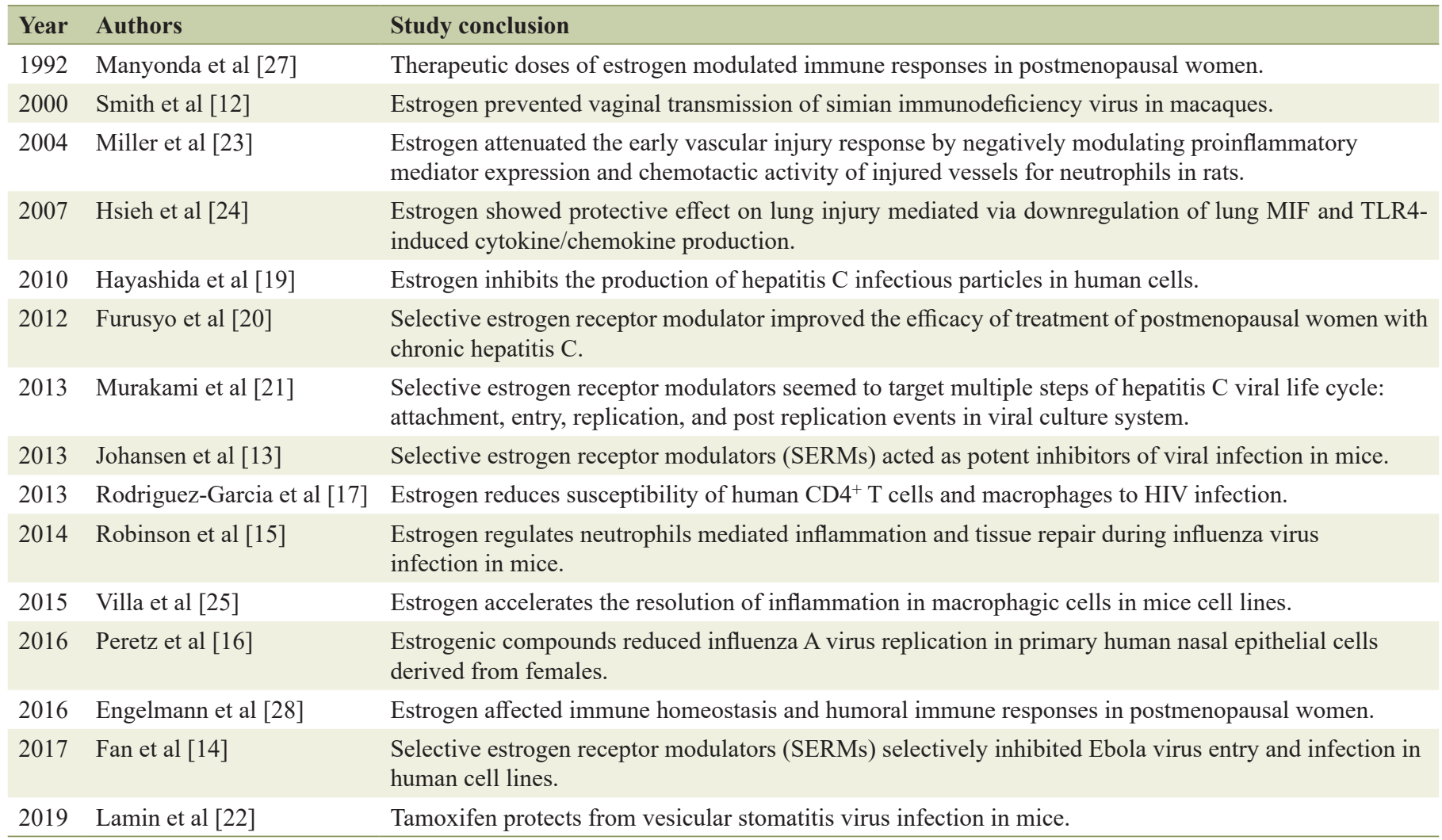

HIV: human immunodeficiency virus.

large proportion of deaths from COVID-19. Estrogen plays a crucial role in the regulation of female cardiovascular health [35], offering protection through repair of endothelial injury and collateral vessel formation in ischemic tissue [36]. The direct actions of estrogen include the increased synthesis and release of endothelia nitric oxide (NO) and prostacyclin vasodilators and anti-platelet agents and reduced production of proinflammatory mediators in situations of vascular injury [36, 37]. While young women generally have much lower rates of cardio-metabolic disease than their age-matched male counterparts, this protection is lost during the menopausal transition [35, 38, 39], when the risks of cardiovascular disease approach and often equal those experienced by men, thus emphasizing the potential role of estrogen. The beneficial effects of hormone replacement therapy (HRT) in preventing atherosclerotic cardiovascular disease are most pronounced when the therapy is initiated before the development of advanced atherosclerosis [36]. A randomized controlled trial showed that the initiation of HRT in women early after menopause significantly reduces the risk of mortality, myocardial infarction or heart failure without resulting in an increased risk of breast cancer or stroke [40]. Starting HRT less than 10 years since a woman's menopause has been shown to lead to a significant reduction in death and a reduction in cardiovascular disease [41], and this has been confirmed in a variety of studies [35, 41]. For women with premature ovarian insufficiency, HRT has ben- eficial effects on plasma lipids, insulin resistance and also on endothelial function, and is recommended at least until the average age of natural menopause (51 years) [39]. Estrogen and its receptors therefore play a key role in health and disease in women. The protection against cardiovascular disease in women of reproductive age is related to estrogen since estrogen levels and receptor expression differ significantly between sexes.

\section{Concluding Remarks}

The gender differences in favor of women in both infection and mortality risk from COVID-19 infection are likely to be multifactorial. While life-style factors could be important, the dominant female hormone estrogen is likely a key player, acting via a variety of mechanisms that include immune-modulation and non-immune mechanisms. The ample evidence from in vitro, animal and human experimentation has been presented above. Perhaps the old adage "man flu" is, after all, far from being just a pejorative term against men to show the superiority of the fairer sex, but actually has a scientific basis [42]. However, our knowledge is far from complete, and further human studies are needed to enable translation into therapeutic approaches. The postmenopausal woman, where estrogen levels have naturally fallen, but can be readily replaced (with HRT), is an ideal human model for further research. 


\section{Acknowledgments}

None to declare.

\section{Financial Disclosure}

No funding was received for this manuscript.

\section{Conflict of Interest}

There are no conflicts of interest to declare. None of the authors have any conflicts of interest to declare.

\section{Author Contributions}

RP and VST wrote the first draft of manuscript. JO and IM revised the draft. All authors approved the final draft of the manuscript.

\section{Data Availability}

The authors declare that data supporting the findings of this study are available within the article (review article only).

\section{References}

1. Gausman J, Langer A. Sex and gender disparities in the COVID-19 pandemic. J Womens Health (Larchmt). 2020;29(4):465-466.

2. WHO. Addressing sex and gender in epidemic-prone infectious diseases. World Health Organization. 2007.

3. Jin JM, Bai P, He W, Wu F, Liu XF, Han DM, Liu S, et al. Gender differences in patients with COVID-19: focus on severity and mortality. Front Public Health. 2020;8:152.

4. Global Health 5050. COVID-19 sex-disaggregated data tracker. Available at: http://globalhealth5050.org/covid19. Accessed Apr 18, 2020.

5. La Vignera S, Cannarella R, Condorelli RA, Torre F, Aversa A, Calogero AE. Sex-specific SARS-CoV-2 mortality: among hormone-modulated ACE2 expression, risk of venous thromboembolism and hypovitaminosis D. Int J Mol Sci. 2020;21(8).

6. Hui DS, Azhar EI, Kim YJ, Memish ZA, Oh MD, Zumla A. Middle East respiratory syndrome coronavirus: risk factors and determinants of primary, household, and nosocomial transmission. Lancet Infect Dis. 2018;18(8):e217e227.

7. Hu Y, Sun J, Dai Z, Deng H, Li X, Huang Q, Wu Y, et al. Prevalence and severity of corona virus disease 2019 (COVID-19): A systematic review and meta-analysis. J Clin Virol. 2020;127:104371.

8. Klein SL, Marriott I, Fish EN. Sex-based differences in immune function and responses to vaccination.
Trans R Soc Trop Med Hyg. 2015;109(1):9-15.

9. Bianchi I, Lleo A, Gershwin ME, Invernizzi P. The X chromosome and immune associated genes. J Autoimmun. 2012;38(2-3):J187-192.

10. Fish EN. The X-files in immunity: sex-based differences predispose immune responses. Nat Rev Immunol. 2008;8(9):737-744.

11. Couse JF, Lindzey J, Grandien K, Gustafsson JA, Korach KS. Tissue distribution and quantitative analysis of estrogen receptor-alpha (ERalpha) and estrogen receptor-beta (ERbeta) messenger ribonucleic acid in the wild-type and ERalpha-knockout mouse. Endocrinology. 1997;138(11):4613-4621.

12. Smith SM, Baskin GB, Marx PA. Estrogen protects against vaginal transmission of simian immunodeficiency virus. J Infect Dis. 2000;182(3):708-715.

13. Johansen LM, Brannan JM, Delos SE, Shoemaker CJ, Stossel A, Lear C, Hoffstrom BG, et al. FDA-approved selective estrogen receptor modulators inhibit Ebola virus infection. Sci Transl Med. 2013;5(190):190ra179.

14. Fan H, Du X, Zhang J, Zheng H, Lu X, Wu Q, Li H, et al. Selective inhibition of Ebola entry with selective estrogen receptor modulators by disrupting the endolysosomal calcium. Sci Rep. 2017;7:41226.

15. Robinson DP, Hall OJ, Nilles TL, Bream JH, Klein SL. 17beta-estradiol protects females against influenza by recruiting neutrophils and increasing virus-specific CD8 T cell responses in the lungs. J Virol. 2014;88(9):47114720.

16. Peretz J, Pekosz A, Lane AP, Klein SL. Estrogenic compounds reduce influenza A virus replication in primary human nasal epithelial cells derived from female, but not male, donors. Am J Physiol Lung Cell Mol Physiol. 2016;310(5):L415-425.

17. Rodriguez-Garcia M, Biswas N, Patel MV, Barr FD, Crist SG, Ochsenbauer C, Fahey JV, et al. Estradiol reduces susceptibility of CD4+ T cells and macrophages to HIVinfection. PLoS One. 2013;8(4):e62069.

18. Straub RH. The complex role of estrogens in inflammation. Endocr Rev. 2007;28(5):521-574.

19. Hayashida K, Shoji I, Deng L, Jiang DP, Ide YH, Hotta H. 17beta-estradiol inhibits the production of infectious particles of hepatitis $\mathrm{C}$ virus. Microbiol Immunol. 2010;54(11):684-690.

20. Furusyo N, Ogawa E, Sudoh M, Murata M, Ihara T, Hayashi T, Ikezaki H, et al. Raloxifene hydrochloride is an adjuvant antiviral treatment of postmenopausal women with chronic hepatitis C: a randomized trial. J Hepatol. 2012;57(6):1186-1192.

21. Murakami Y, Fukasawa M, Kaneko Y, Suzuki T, Wakita T, Fukazawa H. Selective estrogen receptor modulators inhibit hepatitis $\mathrm{C}$ virus infection at multiple steps of the virus life cycle. Microbes Infect. 2013;15(1):45-55.

22. Cham LB, Friedrich SK, Adomati T, Bhat H, Schiller M, Bergerhausen M, Hamdan T, et al. Tamoxifen Protects from Vesicular Stomatitis Virus Infection. Pharmaceuticals (Basel). 2019;12(4).

23. Miller AP, Feng W, Xing D, Weathington NM, Blalock JE, Chen YF, Oparil S. Estrogen modulates inflammatory 
mediator expression and neutrophil chemotaxis in injured arteries. Circulation. 2004;110(12):1664-1669.

24. Hsieh YC, Frink M, Hsieh CH, Choudhry MA, Schwacha MG, Bland KI, Chaudry IH. Downregulation of migration inhibitory factor is critical for estrogen-mediated attenuation of lung tissue damage following trauma-hemorrhage. Am J Physiol Lung Cell Mol Physiol. 2007;292(5):L1227-1232.

25. Villa A, Rizzi N, Vegeto E, Ciana P, Maggi A. Estrogen accelerates the resolution of inflammation in macrophagic cells. Sci Rep. 2015;5:15224.

26. Libert C, Dejager L, Pinheiro I. The X chromosome in immune functions: when a chromosome makes the difference. Nat Rev Immunol. 2010;10(8):594-604.

27. Manyonda IT, Pereira RS, Makinde V, Brincat M, Varma RT. Effect of 17 beta-oestradiol on lymphocyte subpopulations, delayed cutaneous hypersensitivity responses and mixed lymphocyte reactions in post-menopausal women. Maturitas. 1992;14(3):201-210.

28. Engelmann F, Rivera A, Park B, Messerle-Forbes M, Jensen JT, Messaoudi I. Impact of estrogen therapy on lymphocyte homeostasis and the response to seasonal influenza vaccine in post-menopausal women. PLoS One. 2016;11(2):e0149045.

29. Bouman A, Heineman MJ, Faas MM. Sex hormones and the immune response in humans. Hum Reprod Update. 2005;11(4):411-423.

30. Taneja V. Sex hormones determine immune response. Front Immunol. 2018;9:1931.

31. Mohammad I, Starskaia I, Nagy T, Guo J, Yatkin E, Vaananen K, Watford WT, et al. Estrogen receptor alpha contributes to $\mathrm{T}$ cell-mediated autoimmune inflammation by promoting $\mathrm{T}$ cell activation and proliferation. Sci Signal. 2018;11(526).

32. Moulton VR. Sex hormones in acquired immunity and autoimmune disease. Front Immunol. 2018;9:2279.

33. Liao ZH, Huang T, Xiao JW, Gu RC, Ouyang J, Wu G,
Liao H. Estrogen signaling effects on muscle-specific immune responses through controlling the recruitment and function of macrophages and T cells. Skelet Muscle. 2019;9(1):20.

34. Toniolo A, Fadini GP, Tedesco S, Cappellari R, Vegeto E, Maggi A, Avogaro A, et al. Alternative activation of human macrophages is rescued by estrogen treatment in vitro and impaired by menopausal status. J Clin Endocrinol Metab. 2015;100(1):E50-58.

35. Collins P, Webb CM, de Villiers TJ, Stevenson JC, Panay N, Baber RJ. Cardiovascular risk assessment in women an update. Climacteric. 2016;19(4):329-336.

36. Trenti A, Tedesco S, Boscaro C, Trevisi L, Bolego C, Cignarella A. Estrogen, Angiogenesis, Immunity and Cell Metabolism: Solving the Puzzle. Int J Mol Sci. 2018;19(3).

37. Mendelsohn ME, Karas RH. Molecular and cellular basis of cardiovascular gender differences. Science. 2005;308(5728):1583-1587.

38. Baber RJ, Panay N, Fenton A, A. Fenton the IMS Writing Group. 2016 IMS Recommendations on women's midlife health and menopause hormone therapy. Climacteric. 2016;19(2):109-150.

39. Hamoda H, British Menopause S, Women's Health C. The British Menopause Society and Women's Health Concern recommendations on the management of women with premature ovarian insufficiency. Post Reprod Health. 2017;23(1):22-35.

40. Schierbeck LL, Rejnmark L, Tofteng CL, Stilgren L, Eiken P, Mosekilde L, Kober L, et al. Effect of hormone replacement therapy on cardiovascular events in recently postmenopausal women: randomised trial. BMJ. 2012;345:e6409.

41. Menopause: diagnosis and management. NICE. 2015. https://www.nice.org.uk/guidance/ng23.

42. Sue K. The science behind "man flu". BMJ. 2017;359:j5560. 